\title{
Creating a unified Europe: losing identity and visibility for a greater profit?
}

\author{
John F Smyth
}

"No man is an island, entire of itself...if a clod be washed away by the sea, Europe is the less" (John Donne 1571-1631). For "man" and "clod" refer to Society and you are in the midst of a heated debate about the future of oncology in Europe! For the past 20 years the Federation of European Cancer Societies (FECS) has provided a forum for European cancer societies to discuss the development of oncology, promote multidisciplinary management of patients, and share research and educational activities through its flagship biennial ECCO meetings. The six founding societies in FECS (ESMO, ESTRO, ESSO, EACR, EONS and SIOP) were discipline-based, but increasingly the practice of oncology has focused around multidisciplinary organ-based activities. In response to this change, in January 2005 FECS initiated a year-long debate about the future organization of oncology in Europe, recognizing the need to create more dialogue between the older discipline-based societies and the newer organ-based societies that have emerged in recent years. This debate has been constructive and informative but has exposed some strongly held views about the concept of specialist care. As a consequence of these deliberations, FECS proposes to expand its activities by giving full membership to previously 'affiliated' organ-based societies, and to invite new members onto the Board who represent societies involved with major cancer sites (e.g. colorectal, lung, prostate, etc.).

But not everyone is enthusiastic! Some oncologists feel threatened by this move, especially concerning the loss of professional identity; consequently, ESMO has withdrawn from the Federation. Is this paranoia or a genuine desire to ensure excellence in cancer management? Recognition of individual specialties is essential
The need for

individual

specialist

identity should

not detract from

the coherence

of true

multidisciplinary

practice.

JF Smyth is Professor of Medical Oncology at the Cancer Research Centre, University of Edinburgh, UK and President of FECS.

\section{Competing interests}

The author declared he has no competing interests.

www.nature.com/clinicalpractice doi: $10.1038 /$ ncponc0480 to ensure appropriate training and expertise. There is no longer room for the 'generalist' in cancer care, and specialist training and practice is recognized everywhere to provide optimum outcomes. This move towards specialization is a particular challenge in medical oncology, where the development of new medicines with a wider therapeutic index increases the potential for prescribing from nonspecialist oncologists. Indeed, in several European member states medical oncology is not yet recognized as a discreet specialty, and we must all work to establish such recognition. Within the oncology community, however, we must acknowledge the value of teamwork and avoid the concept of any one discipline being superior to another. Multidisciplinary practice and research requires mutual recognition of the contribution that can be made by all specialists from their own particular field. The need for individual specialist identity should not detract from the coherence of true multidisciplinary practice.

FECS is modernizing to provide a panEuropean organization reflecting input from everyone engaged in cancer research and practice, including patients and their representatives. Medical oncology is an essential element in such an organization and many leading European medical oncologists have expressed their wish to be part of FECS. Hopefully, the ESMO Board will reconsider and join the new FECS initiative. The wide variation in the outcomes of cancer treatment across Europe together with differences in member states' health priorities can only be challenged by the political and financial force of such a large pan-European organization. Such a unified force should also help to increase industrial and academic investment in European oncological research. 